\title{
Distributed coding of stimulus magnitude across the rodent prefrontal cortex
}

\author{
Josephine Henke ${ }^{1,2}$, David Bunk ${ }^{1}$, Dina von Werder ${ }^{1}$, Stefan Häusler ${ }^{1,2}$, \\ Virginia L Flanagin ${ }^{2,3}$, Kay Thurley ${ }^{1,2, *}$ \\ ${ }^{1}$ Department Biologie II, Ludwig-Maximilians-Universität München, Germany \\ ${ }^{2}$ Bernstein Center for Computational Neuroscience Munich, Germany \\ ${ }^{3}$ German Center for Vertigo and Balance Disorders, Ludwig-Maximilians-Universität München, Germany \\ * Correspondence: thurley@bio.Imu.de
}

As we interact with the external world, we judge magnitudes from sensory information. The estimation of magnitudes has been characterized in primates, yet it is largely unexplored in non-primate species. Here, we show that gerbils that solve a time-interval reproduction task display primate-like magnitude estimation characteristics, most prominently a systematic overestimation of small stimuli and an underestimation of large stimuli, often referred to as regression effect. We investigated the underlying neural mechanisms by recording from medial prefrontal cortex and show that the majority of neurons respond either during the measurement or the reproduction of a time-interval. Cells that are active during both phases display distinct response patterns. We categorize the neural responses into multiple types and demonstrate that only populations with mixed responses can encode the bias of the regression effect. These results reveal the organizing neural principles of an important higher cognitive function.

Animals including humans estimate the magnitude of physical stimuli, integrate path length and keep track of duration to gather behaviorally relevant information from their environment. Although such estimates may ultimately be used for binary actions, like discriminating items or events and making decisions, the estimation itself is done on a continuum of values. Behavioral analyses over the past century established specific biases in magnitude estimation [e.g. reviewed in 1] such as the regression effect, i.e. the overestimation of small and the underestimation of large stimuli across a range of values. Recently, this bias regained attention as it may be the result of an error minimization strategy $[2-4]$.

Despite a long history of behavioral research on magnitude estimation, its neural basis is not well understood. It is an ongoing debate whether a dedicated or distributed 
magnitude system exists in the brain [for review see 5-7]. Human studies identified frontal, parietal and striatal brain regions that are active during magnitude estimation. Recent studies in non-human primates found that neural population dynamics in frontal and parietal cortices subserve magnitude estimation behavior $[8,9]$. These studies investigated the estimation of time intervals lasting hundreds of milliseconds. It is still unresolved how their findings translate to durations of several seconds, i.e. to time scales that are relevant for more complex and ecologically important behaviors like spatial navigation and action planning. Furthermore, it is unclear how far the results generalize to non-primate species.

We addressed these issues for Mongolian gerbils (Meriones unguiculatus) and designed a psychophysical task for time interval estimation of several seconds on a continuous range. The task was implemented in virtual reality [10], which allows for the precise control of the behaviorally relevant variables.

First, we demonstrate the capability of gerbils to precisely measure and reproduce time intervals of several seconds. We also show that the gerbils' responses display the regression effect, indicative of an error minimization strategy. Then, we present associated neural activity in gerbil medial prefrontal cortex (mPFC). This activity comprises of mixture of responses including phasic activation and ramp-like firing patterns, response types well known from the interval timing literature [e.g. 11-15]. Since our task involves measurement and reproduction, i.e. the timing of an external event and of one's own behavior, we could test how individual cells participated in both. To make the variety of responses tangible, we provide a comprehensive characterization of activity at the single neuron level and show that, despite the response heterogeneity, the mPFC population jointly measures and reproduces time intervals lasting several seconds. We find that distinct types of ramping neurons are necessary to explain the regression effect and thus for a mechanistic understanding of the neural basis of magnitude estimation behavior. This reveals that variables underlying higher cognitive function may be encoded by mixed response types within a local neural population.

\section{Results}

Behavioral characteristics of time reproduction in gerbils. We trained gerbils to measure and reproduce the duration of time intervals lasting a few seconds. After the presentation of a black screen, the animals had to reproduce its duration by walking along a corridor in virtual reality. Intervals were randomly sampled between 3 and $7.5 \mathrm{~s}$. Figure 1a-c details the apparatus and task. Gerbils exhibited typical magnitude estimation behavior [1]. Across the range of stimuli, small stimuli were overestimated whereas large stimuli were underestimated, i.e. the regression effect. This effect was obvious in single sessions (Fig. 1d) and consistent across sessions and animals (slope, Fig. S1a). The average width of the feedback range in a session correlated negatively with the slope of the regression line (Fig. S1c). The data also showed scalar variability, i.e. the standard deviation of reproductions increased with stimulus magnitude (inset in Fig. 1c). Variability (coefficient of variation, Fig. S1a) matched values from other interval timing experiments 
a
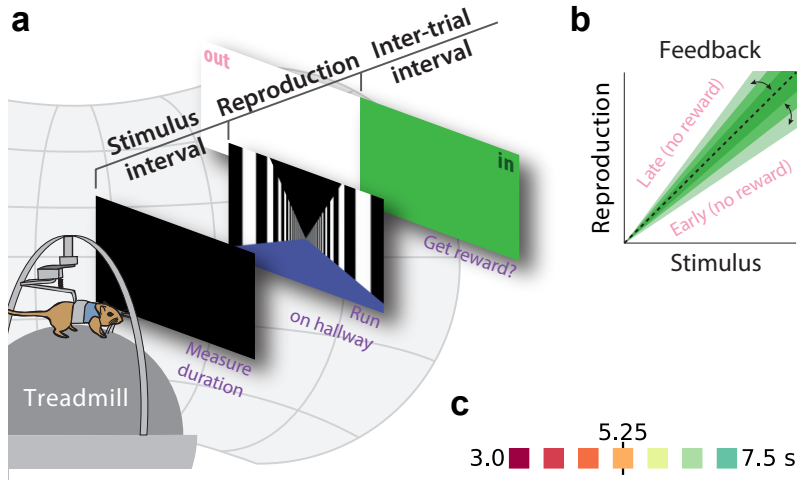

d

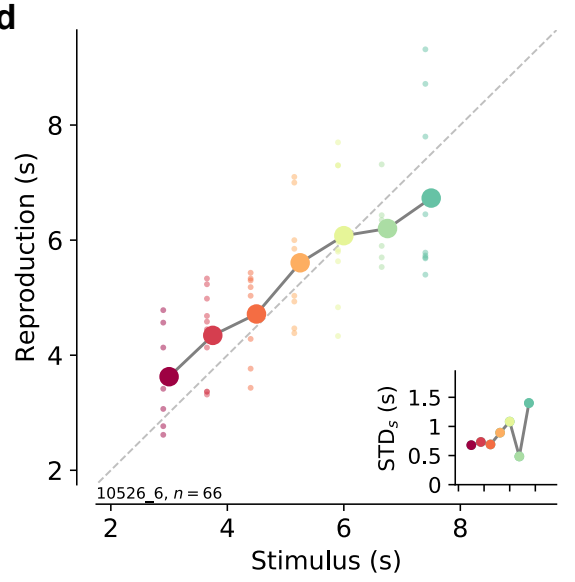

Figure 1. A time reproduction task for rodents. (a) Experimental apparatus and task. A gerbil was placed on top of a spherical treadmill surrounded by a projection screen. Each trial started with a timed stimulus (black screen). The animal had to measure its duration and, when a virtual linear corridor appeared, reproduce this duration by walking. If the reproduction was close to the stimulus ("in", i.e. a hit) a food reward was delivered and the entire screen was set to green for 3-4 seconds before another trial was initiated; for non-hits, screen color was set to white ("out"). (b) The feedback range was reduced/increased after each in/out response. (c) Stimulus intervals were randomly sampled from a discrete uniform distribution with 7 values between 3 and 7.5 s. (d) Behavioral responses exhibited characteristic effects of magnitude estimation. Single reproductions (small dots) and their averages (large connected circles) showed the regression effect (data from one example session). Colors identify stimulus duration and will be used throughout the paper. Inset: Standard deviation increases with stimulus magnitude (scalar variability). The $x$-axis is the same as in the main panel.

and correlated with the strength of regression (STD vs. squared BIAS, Fig. S1b). Some sessions showed a general under- or overestimation in addition to the regression effect (BIAS, Fig. S1a).

Single cells differentially encode time during measurement and reproduction. We recorded a total of $2030 \mathrm{mPFC}$ units over 105 experimental sessions from three gerbils. Visual inspection of the spiking responses, after sorting by stimulus and splitting into the two task phases "measurement" and "reproduction", revealed a variety of response patterns that could underly time reproduction. Three examples are shown in Figure 2, 


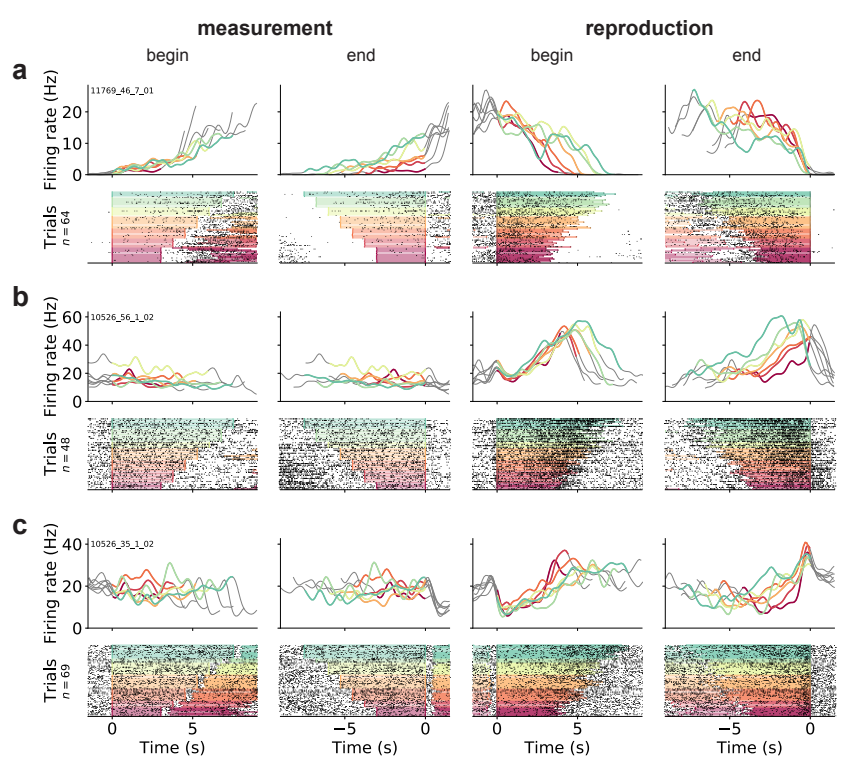

Figure 2. Gerbil mPFC neuron responses during time reproduction. (a) A cell that linearly increased its firing rate during measurement and ramped down to zero during reproduction. (b) A neuron that scaled its firing with the stimulus during reproduction and (c) a ramp-to-threshold cell. (a-c) Panels display spike raster plots sorted by stimulus (bottom) and corresponding spike density functions (SDF, top). Each column plots the data with different alignment, measurement begin and end, reproduction begin and end. Color identifies stimulus as in Fig. 1c. In the raster plots, black ticks are single spikes. For better visualization, we only plot half of the spikes (randomly chosen). Measurement or reproduction phases are delimited by underlayed color. The SDFs are colored in the respective task phase, outside they are displayed as thin gray lines.

further examples can be found in Figures S3-S5.

To represent interval duration, two general response types have been reported: (1) Neurons can encode time by responding phasically at a certain absolute time, e.g. 3 seconds after begin of an interval. Collectively such cells could tile an interval $[14,16,17]$. (2) If their firing rate ramps up or down, neurons can integrate elapsed time $[8,18]$.

During the measurement phase, we only found ramp-like time-dependent firing; no neurons responded with timed peaks. Figure 2 a shows a neuron that ramped up during measurement, such that by the end of the measurement phase its firing rate depended on with the stimulus interval (cf. Fig. S6).

During reproduction, potential timing-related responses were more heterogenous. Neurons, e.g. displayed ramping activity with downward and upward ramps. However, we found two qualitatively different ramp types. Similar to what we saw during measurement, some cells constantly changed their firing rate integrating duration (Fig. 2b). For others, however, the change in firing rate correlated with the stimulus duration such that a fixed firing rate level was reached at the end of reproduction (Fig. 2a\&c and Fig. S6). We also found cells that responded at absolute times (Fig. S4) or relative to the reproduction interval, e.g. at its begin or end (Fig. S5a).

Between measurement and reproduction, the neurons changed their response patterns. We did not observe a single cell that responded in the same way in both task phases. 


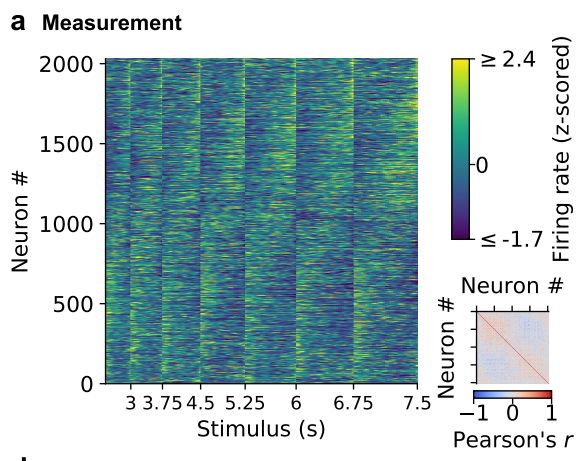

b Reproduction

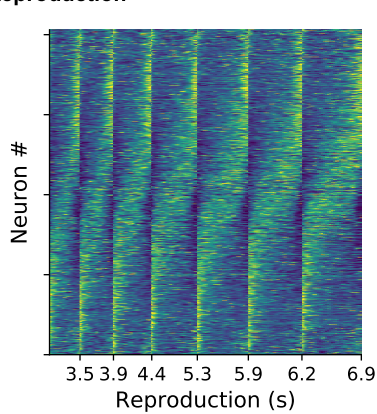

e
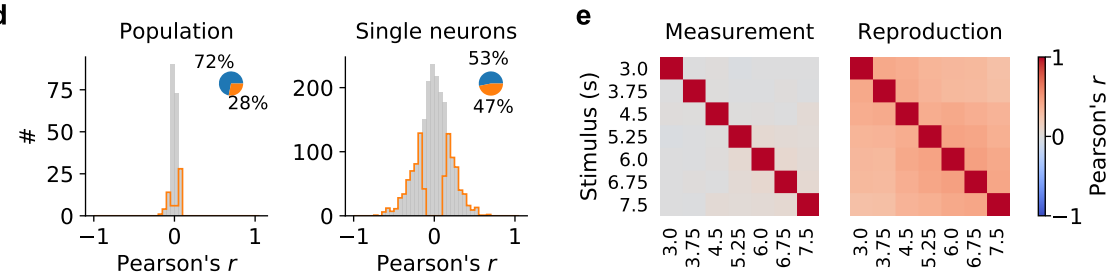
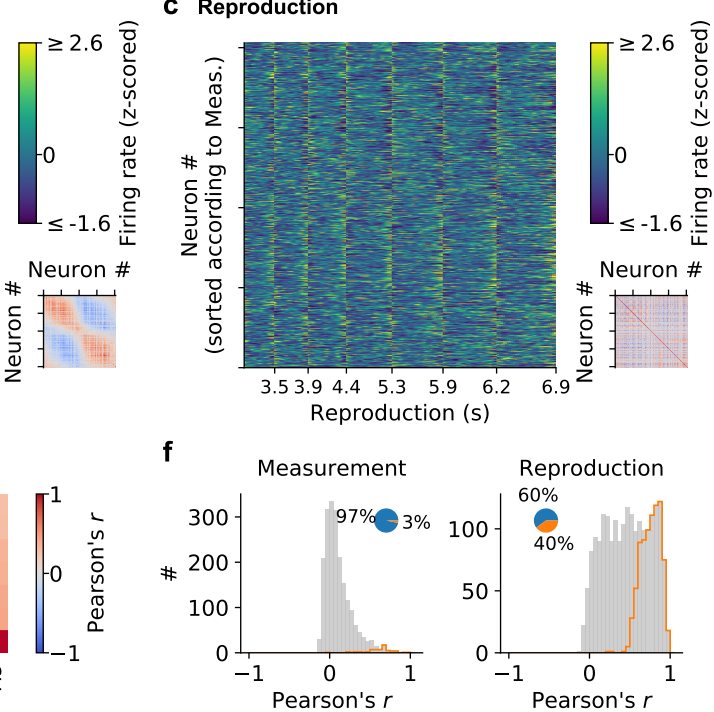

Figure 3. Single neuron and population dynamics in measurement and reproduction. (a) Normalized SDFs of all neurons for each stimulus interval during the measurement phase sorted by their timing within the intervals. Small panel: Matrix of pairwise Pearson correlations between all neurons. (b) Same as (a) but for the reproduction phase. (c) Same as (b) but with neurons sorted as in (a). (d) Distributions of Pearson correlations between measurement and reproduction of the population in each time bin (left) and for single neurons (right). (e) Correlations of the whole population for all stimulus pairs in measurement and reproduction phases. (f) Distributions of average Pearson correlations of single cell activity for different stimuli in measurement and reproduction. Histograms in (d\&f) are displayed in gray with significant values delimited by an orange outline. Pie plots show significant (orange) and non-significant (blue) percentages.

Single cell responses are consistent for the whole population. The different response patterns for single neurons were also obvious when visualizing the whole population. Many of the neurons ramped up at the end of measurement. Other neurons were active at the begin of measurement and then decreased in activity (Fig. 3a). During reproduction, a similar but more pronounced pattern emerged with neurons ramping up and down (Fig. 3b). In addition, some neurons became phasically active in the middle of the interval. Striking, however, were the activity differences between measurement and reproduction, indicating a state change in the population between both task phases. When individual cells were sorted in the same order for both measurement and reproduction, no global pattern was visible (cf. Fig. 3b\&c). Also, correlating population vectors between measurement and reproduction yielded low values (Fig. 3d). In individual cells, however, the correlation between task phases was larger and significant in almost half of the cells (Fig. 3d), indicating that some cells were active during both measurement and reproduction.

Neural activity was similar for different stimulus magnitudes during reproduction. On the one hand, population activity correlated for different stimuli (Fig. 3e); on the other hand, single neuron activity correlated across different stimuli in $40 \%$ of the neurons (Fig. 3f). During measurement, such correlations across stimuli were only weak (Fig. 3e\&f). 

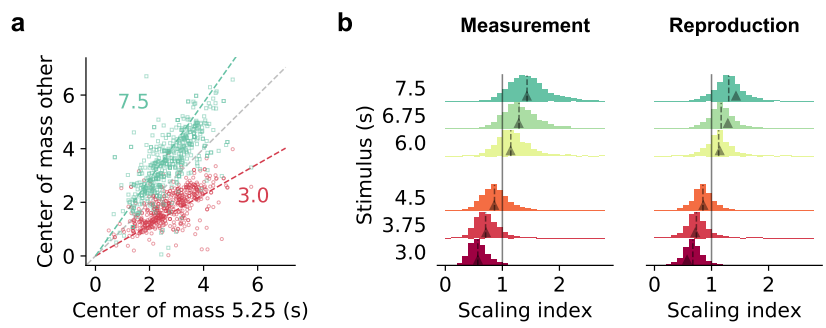

Figure 4. Temporal scaling. (a) Center of mass of each cell for $5.25 \mathrm{~s}$ against 3 (red) and 7.5 (green) during reproduction. Colored dashed lines mark the predicted ratio; gray dashed line corresponds to no change. (b) Distributions of scaling indices for all cells, i.e. center of mass of the SDF at every stimulus divided by the center of mass for 5.25 seconds. Black solid vertical line marks one. Dashed lines mark the medians and arrow heads the ratio between $5.25 \mathrm{~s}$ and the respective other stimulus.

Single neurons scale with duration. The activity of the prefrontal neurons we recorded appeared to scale with stimulus duration (Fig. 3a\&b), which was reminiscent of findings in interval timing studies for striatum [16] and prefrontal cortex [18, 19]. To test for stimulus-dependent scaling, we calculated for each cell the centers of mass of the SDF at every stimulus duration [cf. 16]. The centers of mass we then divided by the value for the $5.25 \mathrm{~s}$ stimulus, i.e. the mean of the stimulus distribution, resulting in scaling indices that approximately range between 0 and 2 .

The scaling indices generally increased for longer stimuli, indicating that SDFs scaled with stimulus duration (Fig. 4). During measurement, the cells' scaling indices were centered over the ratio between the current stimulus duration and $5.25 \mathrm{~s}$, as would be predicted by the stimulus alone. For reproduction, scaling indices were larger or smaller than the prediction in a manner consistent with the regression effect, i.e. smaller durations had larger scaling factors and vice versa.

However, activity lasting the whole task phase but without any meaningful modulation would also yield a stimulus-dependent shift of the center of mass for different stimulus durations. To test whether the scaling was indeed related to an encoding of stimulus duration, we shuffled SDFs (1) across stimuli and cells ("shuffled data") and (2) over time ("noise" controls). During measurement, the distribution of the indices from shuffled data largely matched the original data (Fig. S7a). This was in line with the non-significant correlations of activity across stimuli (Fig. 3e\&f) and indicated no particular temporal scaling during measurement. For the reproduction phase, however, indices from shuffling were more widely distributed and those from noise controls much narrower distributed than the original data (Fig. S7a). To test this further, we determined the correlations between scaling indices and stimulus duration for each cell. During reproduction but not measurement, substantially more single cells showed scaling indices that increased with stimulus magnitude compared to the shuffled data (Fig. S7b). For almost all noise controls, however, scaling indices increased with stimulus, exceeding the situation in the original data.

In particular during reproduction, the centers of mass of the neural responses, but not of the noise controls, tiled the whole time interval (Fig. S8a). Moreover, the correlation 

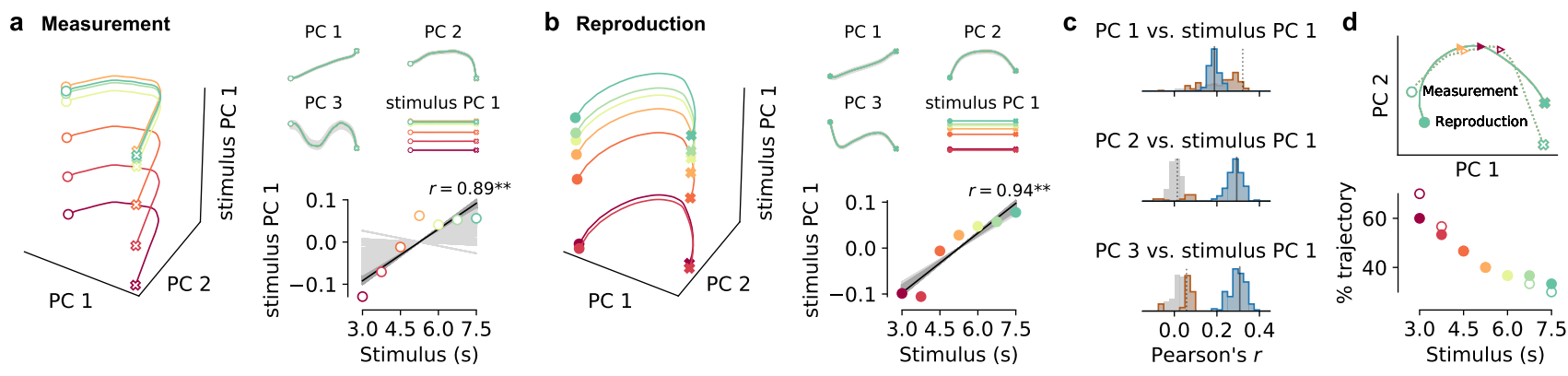

Figure 5. Decomposition of population activity. Demixed PCA yields separate principal components for the time course during a trial (PC 1-3) and for each stimulus (stimulus PC 1). (a) PCs for measurement and (b) for reproduction. Stimuli are colored as in previous figures. Note that PC 1-3 for different stimuli lie on top of each other, demonstrating perfect demixing (compared to conventional PCA, see also Fig. S10). Circles and crosses mark interval start and end. Open symbols are used for measurement and filled for reproduction. The PCs were calculated for the whole data set and gray-shaded areas delimit the standard deviation of results from bootstrapping with $10 \%$ of the cells. Bottom right panels in (a) and (b): Correlation of stimulus PC 1 with the stimulus. Black solid line is a linear fit and gray lines are fits for the bootstrap samples; dark gray significant, light gray non-significant cases. (c) Distributions of Pearson's correlation coefficients between PC 1-3 and stimulus PC 1 from bootstrap samples. Significant correlations are colored in brown (measurement) and blue (reproduction). Dotted (measurement) and solid (reproduction) black lines mark correlations coefficients for the whole data set. (d, upper panel) State-space trajectories for PC 1 and PC 2 in measurement (dashed) and reproduction (solid). Colored triangles mark $2 \mathrm{~s}$ time points for $3.0 \mathrm{~s}$ and $5.25 \mathrm{~s}$ stimuli. (d, lower panel) Percentage of trajectory covered after $2 \mathrm{~s}$ for each stimulus during measurement (open symbols) and reproduction (filled symbols).

coefficients between single cell activity and stimulus duration (Fig. 3f) were larger for neurons with center of mass at the start and end of an interval (Fig. S8b). This suggests that neurons with ramp-like responses scaled their activity with the stimulus. Such a link was only weak during measurement and it did not exist for shuffled controls, noise controls.

Taken together, the above analyses provide evidence for an activity-dependent temporal scaling of prefrontal single cell responses in the reproduction phase alluding to a potential role in time encoding and the regression effect. Scaling during measurement was not different than what would be expected of activity that accompanies elapsing time.

\section{Population activity shares similar components for measurement and reproduction.}

The activity differences between measurement and reproduction revealed by the above analyses argue against a magnitude estimation mechanism solely at the single cell level. Therefore, we examined the population activity for evidence of a common substrate of both measurement and reproduction.

To determine the collective properties of the prefrontal neurons we recorded, we decomposed the population activity into its principal components (PCs). We used demixed PCA a form of principal component analysis (PCA) that allowed us to separate time course-related from stimulus-dependent contributions to neural activity [20].

Despite the dissimilar responses of single cells in measurement and reproduction, the PCs looked very much alike for both task phases (Fig. 5a\&b). The time course-related 
PCs were ramp-like (PC 1) or had a comparable non-monotonous shape (PC 2\&3). Note, however, that PCs 2 and 3 contributed very little to explaining population activity during measurement (Fig. S9). The strongest stimulus PC was constant over time and with amplitudes ordered by stimulus. Moreover, the contribution of the PCs to the single cell responses (PCA scores) correlated for PC 1 and stimulus PC 1 in both measurement and reproduction (Fig. 5c, see also Fig. S11), indicating a link between ramp-like and stimulus-dependent activity. During reproduction, stimulus PC 1 also correlated with PCs 2 and 3, arguing for a richer representation of the to-be-reproduced compared to the to-be-measured time interval.

The collective population representation of time was similar in measurement and reproduction. State-space trajectories overlapped when PC 1 was plotted against PC 2 for both task phases (Fig. 5d). Responses for larger stimuli evolved at lower speed than responses for smaller stimuli [Fig. 5d and Fig. S12; 14, 19]; an effect that corresponds to the temporal scaling described in the previous section.

It is possible that the population responses during measurement and reproduction are simply the result of pooling single neurons and not truly collective phenomena. To test this, we compared population activity to surrogate data that preserve stimulus tuning of single neurons, correlations of single cell firing rates across time and pairwise correlations between neurons [Fig. S13; cf. 21]. During measurement, PCs 1 and 2 of the original data were larger than expected, suggesting that collective activity added to representing ongoing time. Similarly, PCs 2 and 3 were larger than expected during reproduction. Stimulus PC 1 was not different from expectation for reproduction and at the lower end of what was expected during measurement, suggesting that the population as a whole did not add to stimulus representation. Collective activity thus contributed to the representation of ongoing time but not to the representation of the time stimulus.

Heterogeneous prefrontal activity can be categorized into a few response types. To extract response types from a set of neurons, usually, specific tests must be designed based on predefined knowledge of the response of interest. To circumvent such rigid preselection, we used the demixed PCA results for categorizing neurons into different response types (Fig. 5).

Single cell response patterns can be decomposed into linear mixtures of different PCs. We focused at the contributions of the strongest PCs to the single cell responses. During measurement, the two strongest components were time course-related PC 1 and stimulus PC 1 (Fig. S9). Time course-related PC 1 was ramp-like and stimulus PC 1 contained constant responses ordered by stimulus (see Fig. 5a). Mixing both PCs, one could describe responses of cells like the one in Fig. 2a (see also Fig. S3a). For the reproduction phase, time course-related PCs 1-3 and only at forth position stimulus PC 1 were the strongest components. Again, PCs 1-3 displayed time-modulated activity and stimulus PC 1 comprised constants ordered by stimulus. Note furthermore, that - as we already observed above - the PCA scores of single cell responses were correlated between PC 1 and stimulus PC 1 in measurement and PCs 1-3 and stimulus PC 1 in reproduction (Fig. 5c). This points to the potential utility of using these PCs for capturing single cell 
a
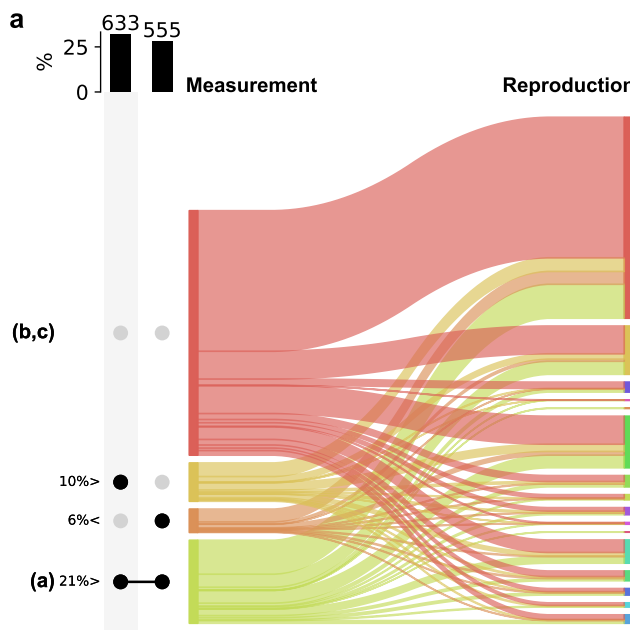

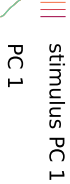

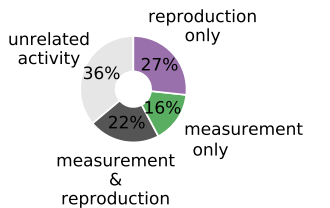

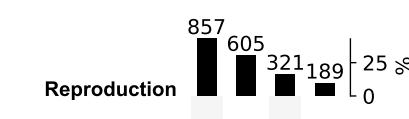

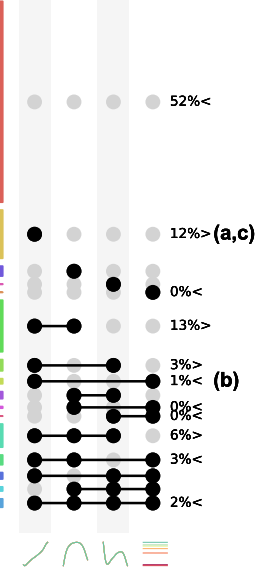

$\stackrel{\overbrace{}}{\longrightarrow}$ ก

$\frac{y}{3}$
3
$\frac{c}{5}$
n
D
$\stackrel{r}{r}$

b Measurement
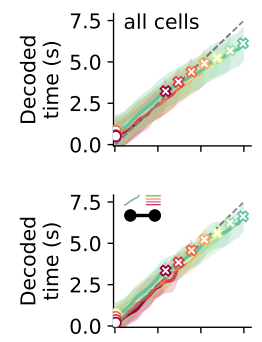

C Reproduction
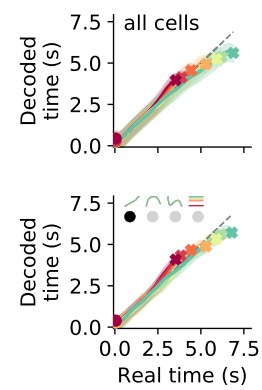

Figure 6. Single cell activity and their contribution to the population response change from measurement to reproduction. (a) The main plot displays the numbers of cells with activity patterns that can be explained by PCs. For measurement (left) only PC 1 and stimulus PC 1 were considered and for reproduction (right) PC 1-3 and stimulus PC 1. Dark dots indicate the contribution of a PC to a category. Percentages are only given for categories that contain significant numbers of cells compared to shuffled data. The signs $<$ and $>$ indicate if the number is smaller or larger than the prediction from shuffling. Zero percentages mean less than $1 \%$. Sankey diagram in the center depicts the flow of cells between categories from measurement to reproduction. Bar graphs at the top are percentages and numbers of cells that can be explained by contributions of one of the PCs. Pie chart at bottom shows percentages of cells active during the task phases determined from the reconstructions displayed in the main plot. Bold letters correspond to the examples cells from Fig. 2. (b\&c) Time representation during measurement is governed by cells that ramp with same slope and finish at a stimulus-dependent level (b) whereas during reproduction the representation is mainly driven by general ramping neurons (c). Decoded elapsed time (avg \pm std from bootstrapping) is shown for the whole population (upper panel) and for cells in stimulus-dependent ramp category (lower panel) for each stimulus interval (color-coded). Circles and crosses mark start and end.

responses.

Using PC 1 and stimulus PC 1 for the measurement phase, three response categories were possible: Activity explained by PC 1 only, by stimulus PC 1 only, or by both PCs. These were divided based on the angle between PC 1 scores and stimulus PC 1 scores (Fig. S11c). Similarly, 15 different categories were defined for the reproduction phase from combinations of PCs 1-3 and stimulus PC 1. We only included cells with large demixed PCA scores (explained variance) in this analysis (Fig. S11). Cells with small scores were categorized as "unrelated activity" adding one more response category.

Dividing cells with small scores from those with large ones, enabled us to estimate the number of cells active during the task phases. Close to a quarter of the cells were active in both phases, another quarter was only active during reproduction, $16 \%$ only during 
measurement and $36 \%$ of the cells did not contribute sufficiently in either phase (pie chart in Fig. 6a).

According to the categorization, during measurement, $21 \%$ of the cells showed ramping activity to stimulus-dependent levels like the neuron in Fig. 2a. Another 6\% represented the stimulus in a different way and in $10 \%$ ramping did not depend on stimulus (Fig. 6a).

During reproduction, $12 \%$ of the neurons showed ramping activity (PC 1 only), e.g. ramp-to-threshold cells where the rate of ramping decreased with stimulus magnitude (c.f. examples in Figs. 2b\&c, S3b\&c). However, a similar fraction was explained by mixing PCs 1 and 2 , about $3 \%$ by PCs 1 and 3, and another $6 \%$ by a mixture of PCs $1-3$. Only $1 \%$ was explained by ramps that encoded stimulus (i.e. PC $1+$ stimulus PC 1 ). However, counting all combinations of stimulus PC 1 with PCs $1 \& 2$ or PCs $1-3$ showed that $6 \%$ of the neurons combined ramps with stimulus-dependent firing. In total, about $42 \%$ of the cells contained a ramping component. These cells overlapped to $81 \%$ with those whose activity was significantly correlated across stimuli (cf. Fig. 3f). In Figures S3-S5 further examples for the different categories can be found.

Cells switched response types between task phases, but no specific pattern of transitions emerged, as obvious from the flow diagram in Fig. 6a.

Time encoding is governed by different response types in both task phases. To determine how the prefrontal neural population encodes elapsed time, we decoded time using simple linear regression. Decoded time was veridical for the first seconds in both task phases. By the end of measurement, decoded times underestimated real time in particular for longer stimuli (Fig. 6b). During reproduction, the regression effect was obvious with over- and underestimation at short and long stimuli, respectively (Fig. 6c). These effects were due to neurons with large PCA scores; cells with small scores generated decoding estimates more similar to shuffled data (Fig. S16). During measurement, time representation was mediated by neurons that ramped to stimulus-dependent levels; whereas time representation was mainly driven by neurons of the ramp-to-threshold type during reproduction (Fig. 6b\&c).

We wondered what response types could actually explain the regression effect and simulated different stereotypical cases (Fig. S17a). Decoding time from neurons that ramp with same slope to stimulus-dependent levels (linear increasing neurons) show veridical time representation but no regression effect. Ramp-to-threshold cells that change slope but reach same activity levels by the end of an interval can only encode the mean of the stimulus distribution and result in regression with slope zero. Phasically active neurons also predict only very strong regression.

We matched response types from our categorization analysis to the theoretical cases and got qualitatively similar results but also specific differences (Fig. S17a). In particular, the ramping neurons in the reproduction phase, i.e. categories comprising neurons that ramp to stimulus-dependent levels and ramp-to-threshold cells, displayed regression effects similar to the behavioral data (Fig. S17c). That both classes show regression may be due to imperfect categorization, but more importantly, it pointed us to a solution to the question how regression effects may be generated: When we combined ramping to 
stimulus-dependent levels with stimulus-dependent slope-modulation (ramp-to-threshold) in single cells or mixed both response types in the presence of noise across the population, the regression effect could be generated (Fig. S17b). Moreover, the strength of regression can be adapted by balancing the fractions of both response types either in individual cells or across a population to capture the different expression seen behaviorally (cf. slope parameter in Fig. S1).

Interestingly, when we only decoded time from ramping cells, a regression effect was also seen during measurement (Fig. S17a), suggesting an impact of previous stimuli (prior knowledge) already on the measurement and not just on the reproduction of a stimulus in magnitude estimation.

\section{Discussion}

We investigated the neural basis of magnitude estimation and analyzed neural correlates from rodent $\mathrm{mPFC}$ in a novel interval timing task. We showed that Mongolian gerbils (Meriones unguiculatus) are able to measure and reproduce durations lasting several seconds. To allow the gerbils to respond in a natural way, we used walking as a response [22]. The task was implemented in a rodent virtual reality system [10], which (1) gave us the use of a treadmill, (2) prevented landmark-based strategies for task-solving and (3) decoupled time from distance, such that the task could not be solved by path integration.

The rodents' behavior exhibited typical characteristics of magnitude estimation, including the regression effect (also known as regression to the mean, central tendency, or Vierordt's law), i.e. the overestimation of small and underestimation of large stimuli [1, 23-25]. Neural activity in gerbil mPFC correlated with and likely contributed to magnitude estimation behavior. Prefrontal neurons displayed various firing characteristics, which could be grouped into a number of representative categories. Single cell firing patterns differed between measurement and reproduction. For those cells that participated in both task phases, their activity profiles, although sometimes correlated, never matched between task phases; see e.g. the ramp-type neurons in Figures S3a,d\&S5a,b. Moreover, activity changes between task phases were not coordinated across neurons, leading to low population vector correlations and no specific transition patterns between response types. Linear decomposition of the population activity, however, revealed state-space trajectories that were only slightly modulated during measurement and reproduction. This indicates that - despite the response heterogeneity within and between cells - the prefrontal population similarly encoded time in both task phases. Such effects on low-dimensional population activity in connection to changes of behavioral and cognitive state have been described for attention and task engagement [26].

Neural correlates of interval timing in the range of seconds have been found in several brain areas [15, 27] including prefrontal cortex [13, 17, 18, 28, 29], pre-/supplementary motor cortex [11, 12], hippocampus [30], entorhinal cortex [31], and striatum [14, 16, 29, 32]. What separates our experiments from previous studies is twofold: (1) We tested time intervals on a continuous range. (2) We combined timing of an external event 
(measurement phase) and timing own behavior (reproduction phase), linking sensory and motor timing [15]. Our study is therefore conceptually different than the fixed interval or discrimination tasks that have been used in most of the above timing studies. Some studies with monkeys used tasks comparable to ours but focused on intervals lasting only hundreds of milliseconds $[8,9]$. The neural activity in primate parietal and frontal cortices observed in these studies is surprisingly similar to what we found in rodent $\mathrm{mPFC}$. This is especially interesting since we tested timing of several seconds and neural dynamics typically act on much shorter time scales.

The $\mathrm{mPFC}$ responses we recorded during the reproduction phase are reminiscent of the neural correlates of self-initiated behavior found in rat secondary motor cortex by Murakami et al. [33, 34]. The reproduction phase in our task also involves self-initiated behavior (i.e. to stop walking). Murakami et al. found ramp-to-threshold cells similar to the one in Figure 2c. However, in contrast to our findings, they reported the absence of such responses in mPFC [34]. This discrepancy may be due to the different tasks involved: waiting for a signal appearing after a random interval in their experiments vs. responding after a previously measured interval in our case. Ramp-to-threshold responses were also reported in monkey pre-/supplementary motor cortices $[11,12]$ and as a population pattern in lateral intraparietal cortex [8] during time (re-)production, demonstrating their ubiquitous presence in self-initiated behaviors. Note, that we also recorded negative ramp-to-threshold, i.e. ramp-down, responses (Fig. 2a).

We also observed linear increasing neurons (neurons that ramp to stimulus-dependent levels at the same slope, e.g. Fig. 2b) that may serve as integrators of time information provided by sequentially-activated, phasically responding cells (Fig. S4). Again, both types of neurons have been reported in other timing tasks [12-14, 35].

Our time decoding analysis revealed that ramp-to-threshold and linear increasing neurons alone are not capable of explaining the regression effect; rather the combination of both response types is necessary in either single neuron activity or mixed across a population of neurons. This view is compatible with theoretical models of interval timing [36] and magnitude estimation [37]. Mixed response types are in general important in cognitive tasks [38] but have also been reported during spontaneous behavior [39]. However, coding of variables related to higher cognitive functions like choice and task engagement is often distributed across brain regions [40]. Although, we only recorded in one brain region and cannot comment on the distribution across the brain, our findings suggest that a local distribution of response types may also underly higher cognitive function.

Temporal scaling appears to be a general feature of timed computations in the brain as it has been described in various brain regions $[16,18,19]$. We found substantial temporal scaling at the single neuron level only in the reproduction phase, likely sustained by ramping neurons. Here, animals had to actively generate timed behavior (motor timing) in contrast to the measurement phase (sensory timing). Still, the speed of collective neural dynamics scaled with the length of the time interval in both measurement and reproduction.

Temporal scaling and speed-dependence of neural dynamics corresponded to the regression effect we observed in the behavioral data. Bayesian models [1, 2] as well as other 
approaches [37, 41] have demonstrated that the regression effect may be a strategy to minimize behavioral errors. Bayesian models fuse probability distributions of the current stimulus estimate and prior knowledge. The neural representations of these probability distributions and the mechanism underlying the probabilistic computations has yet to be determined. An interesting solution was recently proposed based on recordings from monkey frontal cortex by Sohn et al. [9]. While the animals measured time intervals in a task very similar to ours, frontal cortex activity followed low-dimensional curved state-space trajectories. These curved trajectories can be interpreted as a compressed non-linear representation of time, which when read-out appropriately during reproduction, can explain regression effects seen in behavior. Our demixed PCs also showed curved trajectories during measurement (Fig. 5a); however, trajectories from conventional PCA did not comprise such curvatures (Fig. S10a).

Our results point to another solution, namely the regression effect as the consequence of mixed neural responses comprising two different types of ramping. When these types are implemented in different neurons and distributed across the population, the amount of noise in the system determines the strength of the regression effect. Without noise, magnitude encoding would be dominated by neurons with linear increasing activity at constant slope and no regression effect could emerge. If activity is noisy, neurons with stimulus-dependent slope contribute and the regression effect appears. In fact, the amount of noise (uncertainty about the current stimulus) determines the impact of either response type and thus the balance between current stimulus estimate and prior knowledge.

State-space trajectories for different stimuli were well separated during measurement (Fig. 5a). Since stimulus duration is unknown at the beginning of the measurement phase, stimulus-dependent trajectories are likely due to prior expectations about the stimulus. Small stimuli are typically followed by larger ones and vice versa, which may bias neural responses and behavioral estimates accordingly. Such sequential effects are known in the magnitude estimation literature $[1,37,41]$. Interestingly, when we only included ramping cells for time decoding a regression effect was also seen during measurement (Fig. S17a), implying that previous stimuli affect the current measurement. During reproduction, trajectories were also ordered by stimulus (Figs. 5b\&S10b), which is considered an indication that cortical dynamics are adjusted for (re-)producing different time intervals [42].

The present work provides first insight into the neural substrate of magnitude estimation, including the regression effect and error minimization, in rodents. A thorough characterization of $\mathrm{mPFC}$ responses during time-interval reproduction allowed us to show that only mixed responses in either single cells or distributed across a local population of neurons can explain the regression effect. Adjusting the relative fractions of response types one can parameterize the strength of the regression effect and thus the fusion of stimulus estimate and prior knowledge. To resolve the specifics of the underlying neural computations will be an important direction for future research. 


\section{Methods}

\section{Animals}

The experiments in this study were conducted with three female adult Mongolian gerbils (Meriones unguiculatus) from a wild-type colony at the local animal house (referred to by ids 10526, 11769, and 11770). Training started at an age of at least four months. The gerbils were housed individually on a 12-h light/dark cycle, and all behavioral training and recording sessions were performed in the light phase of the cycle. The animals received a diet maintaining them at about $85-95 \%$ of their free feeding weight. All experiments were approved according to national and European guidelines on animal welfare (Reg. von Oberbayern, AZ 55.2-1-54-2532-10-11 and AZ 55.2-1-54-2532-70-2016).

\section{Behavioral experiments}

Experimental apparatus. Experiments were done on a virtual reality (VR) setup for rodents (Fig. 1a). For a detailed description see Thurley et al. [43]. In brief, the setup consists of an air-suspended styrofoam sphere that acts as a treadmill. On top of the sphere the rodent is fixated with a harness that leaves head and legs freely movable. Rotations of the sphere are induced when the animal moves its legs. The rotations are detected by infrared sensors and fed into a computer to generate and update a visual virtual scene. The scene is displayed via a projector onto a projection screen that surrounds the treadmill. We used Vizard Virtual Reality Toolkit (v5, WorldViz, http: //www . worldviz.com) for real-time rendering; the virtual environment was designed with Blender (v2.49b, http://www.blender.org/). Animals were rewarded with food pellets $(20 \mathrm{mg}$ Purified Rodent Tablet, banana \& chocolate flavor, TestDiet, Sandown Scientific, UK) that were automatically delivered and controlled by the VR software.

Behavioral paradigm. In our interval reproduction task, a rodent had to estimate the duration of a visual stimulus and reproduce it by moving along a virtual corridor. It is thus a variant of the "ready-set-go" timing task by Jazayeri \& Shadlen [2]. Figure 1a illustrates the basic procedure: Each trial started with the presentation of a temporal stimulus - a black screen. Animals were trained to measure its duration and not to move during this phase of the task. Stimuli were randomly chosen between 3 and 7.5 seconds (i.e. either $3,3.75,4.5,5.25,6,6.75$, or $7.5 \mathrm{~s}$ ). Afterwards, the visual scene switched, a virtual corridor appeared and the animal had to reproduce the stimulus by moving through the corridor for the same duration. The animal decided on its own when to start reproducing the interval as well as when to stop. Continuous movement for at least $1 \mathrm{~s}$ was necessary to start reproduction; stopping for more than $0.5 \mathrm{~s}$ was counted as the end of reproduction. With this procedure, we avoided counting brief movements and stops as responses.

We gave feedback to our gerbils on their reproduction performance. Following the reproduction phase, the entire projection screen was either set to green (positive, "in" or "hit") or white (negative, "out") for 3-4 s. In addition, the animal was rewarded for a 
hit with one food pellet. For a hit, the reproduction had to be sufficiently close to the stimulus interval, i.e. $(1 \pm k) \times$ stimulus. The width of this feedback range depended on the stimulus since errors increase with stimulus, i.e. scalar variability. Across the session, tolerance $k$ was reduced by $-3 \%$ for hits and extended by $+3 \%$ otherwise (Fig. 1b). In the first trial of a session, $k$ was always set to the value from the last trial in the previous session. Adapting $k$ over a session, animals reached values close to $15 \%$ and below on average, and hit rates between $50 \%$ and $75 \%$ (Fig. S1a).

The virtual corridor was designed to exclude landmark-based strategies. It was infinite and had a width of $0.5 \mathrm{~m}$. The walls of $0.5 \mathrm{~m}$ height were covered with a repetitive pattern of black and white stripes, each with a height to width ratio of 1:5. The floor was homogeneously colored in medium light-blue and the sky was black.

By randomly changing the gain between an animals' own-movement (i.e. movement of the treadmill) and movement in VR, we de-correlated movement time from virtual distance and thus prevented path integration strategies for task solving. Gain values were uniformly sampled between 0.25 and 2.25 .

Behavioral training and testing. Naive gerbils were accustomed to the VR setup in a virtual linear maze for five to ten sessions [ $\sim 2$ weeks, cf. 43]. Then, we exposed the animals to the timing task. As a first step, we presented only stimuli of 3 and $6 \mathrm{~s}$ which were easy to distinguish for the animals. The animals had to learn to either walk for a short or a long duration. Feedback was initially given with a tolerance of $k=50 \%$ and training proceeded until values below $30 \%$ were reached for at least three subsequent sessions. This training phase took about 1.5 months (about 30 sessions). In the second part of the training, we presented the full stimulus range for about 7 sessions (1.5 weeks), to introduce the animals to stimuli on a continuous scale. Afterwards, we implanted tetrodes into the animals' $\mathrm{mPFC}$ and continued with the test phase.

Analysis of behavioral data. To compare behavioral performance across sessions and animals, we calculated different measures. To quantify the strength of the regression effect, we determined the slope of the linear regression between stimulus $s$ and reproduction $r$. A slope of one would correspond to no regression and smaller slopes to stronger regression. Indifference points were calculated from the slope and intercept of the regression line as IDP $=\frac{\text { intercept }}{1-\text { slope }}$. The mean squared error (MSE) gives the deviation between stimulus $s$ and reproduction $r \operatorname{MSE}(r)=\mathrm{E}\left[(r-s)^{2}\right]$. It can be split into two contributions

$$
\begin{aligned}
\operatorname{MSE}(r) & =\mathrm{E}_{s}\left[\operatorname{Var}_{s}(r)\right]+\mathrm{E}_{s}\left[\operatorname{BIAS}_{s}^{2}(r)\right] \\
& =\underbrace{\mathrm{E}_{s}\left[\mathrm{E}_{r}\left[\left(r-\mathrm{E}_{r}[r \mid s]\right)^{2} \mid s\right]\right]}_{\operatorname{Var}(r)}+\underbrace{\mathrm{E}_{s}\left[\left(\mathrm{E}_{r}[r \mid s]-s\right)^{2}\right]}_{\operatorname{BIAS}^{2}(r)},
\end{aligned}
$$

where $\operatorname{Var}_{s}(r)$ is the variance and $\operatorname{BIAS}^{2}(r)$ the squared bias of the responses for stimulus $s$, and $\mathrm{E}_{s}[\cdot]$ and $\mathrm{E}_{r}[\cdot]$ denote expected values over stimuli $s$ or responses $r$. Variability is often measured by the $\mathrm{CV}$, which we calculated as $\mathrm{CV}(r)=\mathrm{E}_{s}\left[\frac{\mathrm{STD}_{s}(r)}{\mathrm{E}_{r}[r \mid s]}\right]$. To quantify general under- or overestimation, we use the signed $\operatorname{BIAS}(r)=\mathrm{E}_{s}\left[\mathrm{E}_{r}[r \mid s]-s\right]$. 


\section{Electrophysiological recordings}

Electrode implantation. We chronically implanted gerbils with 8 tetrodes mounted to an microdrive that allowed for movement of all tetrodes together (Axona Ltd., St. Albans, UK). Tetrodes were made of $17 \mu \mathrm{m}$ platinum-iridium wires (California Fine Wire Co.). For surgery, we anesthetized an animal with an initial dose of medetomidine-midazolamfentanyl $(0.15 \mathrm{mg} / \mathrm{kg}, 7.5 \mathrm{mg} / \mathrm{kg}, 0.03 \mathrm{mg} / \mathrm{kg}$, s.c.) and later maintained anesthesia by $2 / 3$ doses every $2 \mathrm{~h}$. The animal was placed on a heating pad to keep body temperature at $37^{\circ} \mathrm{C}$ and fixated in a stereotactic unit (Stoelting Co.). After giving local analgesia of the skull with lidocaine (Xylocain, Astra Zeneca $\mathrm{GmbH}$ ), we drilled a hole into the skull above the right $\mathrm{mPFC}$ and placed tetrodes at an initial depth of $700 \mu \mathrm{m}$ into the cortex [2.1 mm AP, $-0.7 \mathrm{~mm} \mathrm{ML},-0.7 \mathrm{~mm} \mathrm{DV} ; 44]$. To protect the exposed part of the brain, we used alginate (0.5\% sodium alginate and $10 \%$ calcium chloride, Sigma-Aldrich) and paraffin wax. Further holes were drilled into the frontal, parietal and occipital bone to place small jewelers' screws to help anchoring the microdrive to the skull with dental acrylic (iBond Etch, Heraeus Kulzer GmbH, Germany; Simplex Rapid, Kemdent, UK). One of the screws served as electrical ground. At the end of the surgery, anesthesia was antagonized with atipamezole-flumazenil-naloxone $(0.4 \mathrm{mg} / \mathrm{kg}, 0.4 \mathrm{mg} / \mathrm{kg}, 0.5 \mathrm{mg} / \mathrm{kg}$, s.c.). During surgery and for three postsurgical days, we gave meloxicam as a painkiller $(0.2$ $\mathrm{mg} / \mathrm{kg}$, s.c.). In addition, enrofloxacin antibiosis (Baytril, $10 \mathrm{mg} / \mathrm{kg}$, s.c.) was done for five to seven postsurgical days. The animals were allowed to recover for at least three days after surgery before recordings started.

Recording procedures. Extracellular action potentials of single units were recorded at a rate of $32 \mathrm{kHz}$ (Digital Lynx SX, Neuralynx, Inc.). Unit activity was band-pass filtered at $600 \mathrm{~Hz}$ to $6 \mathrm{kHz}$. Each tetrode could be recorded differentially, being referenced by one electrode of another tetrode or the ground connected to one of the jewelers' screws. Recordings were done with Neuralynx' data acquisition software Cheetah v5.6.3.

To sample of different neurons throughout the experimental period, we lowered the position of the tetrodes along the dorsoventral axis of the medial prefrontal cortex. Lowering was done for $50 \mu \mathrm{m}$ at the end of every second experimental session to allow for stabilization until the next experiment.

Reconstruction of tetrode placement. Tetrode placement was verified histologically postmortem. Animals received an overdose of sodium pentobarbital and were perfused intracardially with $4 \%$ paraformaldehyde. Brains were extracted and incubated in paraformaldehyde for 1-2 days. Afterwards, the brain was washed in $0.02 \mathrm{M}$ phosphate buffered saline and coronal slices of 60-80 $\mu \mathrm{m}$ thickness were obtained and stained either with Neutralred or DiI (D282), NeuroTrace 500/525 Green Fluorescent Nissl Stain, and DAPI - all stains from Thermo Fischer Scientific. Histology of all animals can be found in Figure S2a. 


\section{Analysis of electrophysiological data}

A total of 2030 mPFC neurons were recorded over 105 experimental sessions, each with on average more than 50 trials (Fig. S1d); animal 10526: 415 cells in 40 sessions; animal 11769: 737 cells in 32 sessions; and animal 11770: 878 cells in 33 sessions.

Spike sorting. Spike sorting was done offline in two steps. First, data was automatically clustered with KlustaKwik (v1.6). Afterwards, clusters were improved manually in 2D projections of waveform features incl. peak and valley, the difference between both, and energy, i.e. integral of the absolute value of the waveform, with MClust v4.3 (http:// redishlab.neuroscience. umn.edu/MClust/MClust.html) under MATLAB2015b (The MathWorks, Inc.).

Only units with stable firing throughout a session entered further analysis. A unit was considered stable if spike counts in 1 min windows did not drop below four standard deviations from the mean session firing rate.

Spike density functions (SDFs). We calculated SDFs for each task phase separately. To calculate a SDF, spikes were either aligned at the begin or the end of the task phase. Then, counts were determined in $100 \mathrm{~ms}$ windows for all trials at a stimulus and divided by window width to gain firing rates. Finally, the SDF was smoothed with a Gaussian kernel with 3-bin standard deviation.

For the analyses in Figures 3-6 and accompanying supplementary figures, SDFs were zscored to account for cell-specific differences in firing rate. In addition, we time-normalized (resampled to same length) the SDFs of different cells and for different stimuli, to be able to compare data from stimuli and responses of different duration.

For the population plots in Figure 3a-c, neurons were sorted by the angle between their demixed PCA scores for the first two time course-related PCs (see below for the description of the demixed PCA). This takes into account the full response profile instead of single features like the peak firing rate.

Control SDFs used in Figures S7, S8, and S16 were generated by (1) shuffling SDFs across stimuli and cells, i.e. "shuffled data", and (2) shuffling single responses over time, i.e. "noise".

Single cell and population correlations. In Figure 3 we determined several different Pearson correlations of the single cell and population data. All correlations were calculated on the time-normalized SDFs. Pairwise correlations were determined for the responses to all stimuli between all pairs of neurons (insets in Fig. 3a-c). The population vector correlation was calculated as the activity of all neurons in one time bin during measurement and the corresponding time bin during reproduction (Fig. 3d, left). Single cell activity was correlated for all stimuli between measurement and reproduction (Fig. 3d, right). In Figure 3e, pairwise correlations were calculated between the responses of the whole population for all stimuli. Similarly pairwise correlations were determined for each individual cell in Figure 3f. 
Principal component analysis (PCA). Demixed PCA [20] was performed separately for measurement and reproduction on the SDFs of all recorded neurons aligned at the respective onsets (Fig. 5). We used the demixed PCA implementation available at https://github.com/machenslab/dPCA. When we applied demixed PCA on data from individual animals results were similar (Figs. S9, S14, and S15). Normal PCA was also done separately for measurement and reproduction and on the SDFs of all recorded neurons aligned at the respective onsets (Fig. S10).

Bootstrapping was done by performing demixed PCA on 1000 random subsets comprising each $10 \%$ of the whole data set, i.e. 203 neurons. Subsets were picked in a stratified way, i.e. accounting for the different numbers of cells recorded in each animal. The function StratifiedShuffleSplit from Scikit-learn was used for picking the subsets. Results were similar for $5 \%$ and $20 \%$ subsets.

Tensor maximum entropy surrogates. To test whether population responses contained collective contributions beyond what is expected from pooling single neurons, we generated control surrogate data according to [21]. Random tensor maximum entropy surrogate samples were drawn that preserved the stimulus tuning of single neurons, correlations of single cell firing rates across time and signal correlations across neurons. The implementation we used is available at https://github.com/gamaleldin/rand_tensor.

Categorization of response types. We categorized cells into different response types by their score values for specific principal components: time course-related PC 1 and stimulus PC 1 for measurement and time course-related PCs 1-3 and stimulus PC 1 for reproduction. Since the scores for those PCs had single peaked distributions, displaying no obvious clusters or response groups (Fig. S11), we used the following procedure to construct response categories: Cells with an explained variance below the cumulative overall explained variance for the PCs (measurement: 14\%, reproduction: 45\%; cf. Fig. S9) were sorted into the unrelated activity category; cf. Fig. S11. For all other cells, first the strongest PC (the one with the largest absolute scores) was determined and then the angles between this PC and each of the other PCs were determined (calculated on the absolute values). If any of those angles was above $22.5^{\circ}$, the other component was counted as contributing as well (cf. Fig. S11c). In total, $2^{2}=4$ different response types were possible in the measurement phase and $2^{4}=16$ in the reproduction phase, i.e. categories ranging from "unrelated activity" with no overlap with any of the PCs to activity explained by all PCs used for categorization.

Categories were validated by categorizing every cell by its scores for each of the 1000 bootstrapped demixed PCAs described above. Finally, the category with maximum likelihood was assigned to the cell.

In addition, we compared the number of cells in each category to a random prediction. For that, we constructed random surrogate data by shuffling SDFs across stimuli and cells, performed demixed PCA on this data, categorized each "surrogate cell" and counted the number of cells in each category. From 1000 such shufflings we got distributions of by chance expected cell counts in each category, which we used to determine $p$-values for 
the count in the original data. A level of $5 \%$ was chosen and indicated as significant in Figure 6.

Time decoding. To decode elapsed time we used multiple linear regression [Wiener filter; 45, https : //github.com/KordingLab/Neural_Decoding] between time bins and the corresponding spike rate (SDF) for every neuron and at each stimulus. We first fit the whole data set and then decoded times from the responses at each stimulus individually. This treated the whole data set as a reference (prior) to which the activity at a particular stimulus was related. Fitting and decoding was performed on random subsets of 20 cells (2000 bootstrap runs), from which we extracted average and standard deviation.

\section{Additional notes on data analysis}

Data analysis was done with Python 2.7 using - in addition to above mentioned packages - Matplotlib 2.2, Numpy 1.15, Pandas 0.24, Scipy 1.2, Scikit-learn 0.20, and Statsmodels 0.10 . If $p$-values are not provided, significance is indicated by ${ }^{*} p<0.05 ;{ }^{* *} p<0.01$; ${ }^{* * *} p<0.001$.

\section{Acknowledgments}

We thank Tobias Bernklau for help with data analysis and Moritz Dittmeyer for help with the schematic drawing of the setup. This work was supported by BMBF (Federal Ministry of Education and Research, Germany) via Bernstein Center Munich (grant number 01GQ1004A).

\section{Author contributions}

KT and VFL envisioned the study and designed the behavioral paradigm. JH and KT designed and performed the experiments. KT, JH, DB, and DvW analyzed the data. KT and $\mathrm{SH}$ developed the method for categorization of response types. KT wrote the paper with help from the other authors.

\section{Competing interests}

The authors declare no competing financial interests.

\section{Data availability}

The datasets generated and analyzed in this study are available from the corresponding author upon reasonable request. 


\section{Code availability}

The code used for analysis in this study is available from the corresponding author upon reasonable request.

\section{References}

1. Petzschner, F. H., Glasauer, S. \& Stephan, K. E. A Bayesian perspective on magnitude estimation. Trends Cogn Sci 19, 285-293 (2015).

2. Jazayeri, M. \& Shadlen, M. N. Temporal context calibrates interval timing. Nat Neurosci 13, 1020-1026 (2010).

3. Petzschner, F. H. \& Glasauer, S. Iterative Bayesian estimation as an explanation for range and regression effects: a study on human path integration. J Neurosci $\mathbf{3 1}$, 17220-17229 (2011).

4. Cicchini, G. M., Arrighi, R., Cecchetti, L., Giusti, M. \& Burr, D. C. Optimal encoding of interval timing in expert percussionists. J Neurosci 32, 1056-1060 (2012).

5. Kadosh, R. C., Lammertyn, J. \& Izard, V. Are numbers special? An overview of chronometric, neuroimaging, developmental and comparative studies of magnitude representation. Progress in Neurobiology 84, 132-147 (2008).

6. Bueti, D. \& Walsh, V. The parietal cortex and the representation of time, space, number and other magnitudes. Philosophical Transactions of the Royal Society B: Biological Sciences 364, 1831-1840 (2009).

7. Van Opstal, F. \& Verguts, T. Is there a generalized magnitude system in the brain? Behavioral, neuroimaging, and computational evidence. Frontiers in Psychology 4, 435 (2013).

8. Jazayeri, M. \& Shadlen, M. N. A Neural Mechanism for Sensing and Reproducing a Time Interval. Curr Biol 25, 2599-609 (2015).

9. Sohn, H., Narain, D., Meirhaeghe, N. \& Jazayeri, M. Bayesian Computation through Cortical Latent Dynamics. Neuron 103, 934-947.e5 (2019).

10. Thurley, K. \& Ayaz, A. Virtual reality systems for rodents. Curr Zool 63, 109-119 (2017).

11. Mita, A., Mushiake, H., Shima, K., Matsuzaka, Y. \& Tanji, J. Interval time coding by neurons in the presupplementary and supplementary motor areas. Nat Neurosci 12, 502-507 (2009).

12. Merchant, H., Zarco, W., Perez, O., Prado, L. \& Bartolo, R. Measuring time with different neural chronometers during a synchronization-continuation task. PNAS 108, 19784-19789 (2011).

13. Kim, J., Ghim, J.-W., Lee, J. H. \& Jung, M. W. Neural correlates of interval timing in rodent prefrontal cortex. J Neurosci 33, 13834-13847 (2013). 
14. Gouvêa, T. S. et al. Striatal dynamics explain duration judgments. eLife 4 (2015).

15. Paton, J. J. \& Buonomano, D. V. The Neural Basis of Timing: Distributed Mechanisms for Diverse Functions. Neuron 98, 687-705 (2018).

16. Mello, G. B. M., Soares, S. \& Paton, J. J. A scalable population code for time in the striatum. Curr Biol 25, 1113-1122 (2015).

17. Tiganj, Z., Jung, M. W., Kim, J. \& Howard, M. W. Sequential Firing Codes for Time in Rodent Medial Prefrontal Cortex. Cereb Cortex 27, 5663-5671 (2017).

18. Xu, M., Zhang, S.-y., Dan, Y. \& Poo, M.-i. Representation of interval timing by temporally scalable firing patterns in rat prefrontal cortex. PNAS 111, 480-485 (2014).

19. Wang, J., Narain, D., Hosseini, E. A. \& Jazayeri, M. Flexible timing by temporal scaling of cortical responses. Nat Neurosci 21, 102-110 (2018).

20. Kobak, D. et al. Demixed principal component analysis of neural population data. eLife 5 (2016).

21. Elsayed, G. F. \& Cunningham, J. P. Structure in neural population recordings: an expected byproduct of simpler phenomena? Nat Neurosci 20, 1310-1318 (2017).

22. Meijer, J. H. \& Robbers, Y. Wheel running in the wild. Proc. R. Soc. B 281 (2014).

23. Von Vierordt, K. Der Zeitsinn nach Versuchen (Laupp, Tübingen, 1868).

24. Hollingworth, H. L. The central tendency of judgment. The Journal of Philosophy, Psychology and Scientific Methods, 461-469 (1910).

25. Shi, Z., Church, R. M. \& Meck, W. H. Bayesian optimization of time perception. Trends Cogn Sci (2013).

26. Engel, T. A. \& Steinmetz, N. A. New perspectives on dimensionality and variability from large-scale cortical dynamics. Current Opinion in Neurobiology 58, 181-190 (2019).

27. Merchant, H., Harrington, D. L. \& Meck, W. H. Neural basis of the perception and estimation of time. Annu Rev Neurosci 36, 313-336 (2013).

28. Genovesio, A., Tsujimoto, S. \& Wise, S. P. Neuronal activity related to elapsed time in prefrontal cortex. J Neurophysiol 95, 3281-3285 (2006).

29. Emmons, E. B. et al. Rodent medial frontal control of temporal processing in the dorsomedial striatum. J Neurosci (2017).

30. MacDonald, C. J., Lepage, K. Q., Eden, U. T. \& Eichenbaum, H. Hippocampal "time cells" bridge the gap in memory for discontiguous events. Neuron 71, 737-749 (2011).

31. Heys, J. G. \& Dombeck, D. A. Evidence for a subcircuit in medial entorhinal cortex representing elapsed time during immobility. Nat Neurosci 21, 1574-1582 (2018).

32. Bakhurin, K. I. et al. Differential Encoding of Time by Prefrontal and Striatal Network Dynamics. J Neurosci 37, 854-870 (2017). 
33. Murakami, M., Vicente, M. I., Costa, G. M. \& Mainen, Z. F. Neural antecedents of self-initiated actions in secondary motor cortex. Nat Neurosci 17, 1574-1582 (2014).

34. Murakami, M., Shteingart, H., Loewenstein, Y. \& Mainen, Z. F. Distinct Sources of Deterministic and Stochastic Components of Action Timing Decisions in Rodent Frontal Cortex. Neuron 94, 908-919.e7 (2017).

35. Genovesio, A., Seitz, L. K., Tsujimoto, S. \& Wise, S. P. Context-Dependent Duration Signals in the Primate Prefrontal Cortex. Cereb Cortex 26, 3345-3356 (2016).

36. Simen, P., Balci, F., de Souza, L., Cohen, J. D. \& Holmes, P. A model of interval timing by neural integration. J Neurosci 31, 9238-9253 (2011).

37. Thurley, K. Magnitude estimation with noisy integrators linked by an adaptive reference. Front Integr Neurosci 10, 6 (2016).

38. Rigotti, M. et al. The importance of mixed selectivity in complex cognitive tasks. Nature 497, 585-590 (2013).

39. Stringer, C. et al. Spontaneous behaviors drive multidimensional, brainwide activity. Science 364, 255 (2019).

40. Steinmetz, N. A., Zatka-Haas, P., Carandini, M. \& Harris, K. D. Distributed coding of choice, action and engagement across the mouse brain. Nature 576, 266-273 (2019).

41. Bausenhart, K. M., Dyjas, O. \& Ulrich, R. Temporal reproductions are influenced by an internal reference: explaining the Vierordt effect. Acta Psychol (Amst) 147, 60-67 (2014).

42. Remington, E. D., Narain, D., Hosseini, E. \& Jazayeri, M. Flexible sensorimotor computations through rapid reconfiguration of cortical dynamics. Neuron (2018).

43. Thurley, K. et al. Mongolian gerbils learn to navigate in complex virtual spaces. Behav Brain Res 266, 161-168 (2014).

44. Radtke-Schuller, S. et al. Brain atlas of the Mongolian gerbil (Meriones unguiculatus) in CT/MRI-aided stereotaxic coordinates. Brain Structure \& Function 221 Suppl 1, 1-272 (2016).

45. Glaser, J. I., Chowdhury, R. H., Perich, M. G., Miller, L. E. \& Kording, K. P. Machine learning for neural decoding. arXiv preprint arXiv:1708.00909 (2017). 